\title{
A novel point mutation in RpoB improves osmotolerance and succinic acid production in Escherichia coli
}

\author{
Mengyong Xiao ${ }^{1,2,3+}$, Xinna Zhu ${ }^{1,2+}$, Hongtao Xu ${ }^{1,2}$, Jinlei Tang ${ }^{1,2}$, Ru Liu ${ }^{1,2}$, Changhao Bi ${ }^{1,2}$, Feiyu Fan ${ }^{1,2^{*}}$
} and Xueli Zhang ${ }^{1,2^{*}}$

\begin{abstract}
Background: Escherichia coli suffer from osmotic stress during succinic acid (SA) production, which reduces the performance of this microbial factory.

Results: Here, we report that a point mutation leading to a single amino acid change (D654Y) within the $\beta$-subunit of DNA-dependent RNA polymerase (RpoB) significantly improved the osmotolerance of E. coli. Importation of the D654Y mutation of RpoB into the parental strain, Suc-T110, increased cell growth and SA production by more than $40 \%$ compared to that of the control under high glucose osmolality. The transcriptome profile, determined by RNA-sequencing, showed two distinct stress responses elicited by the mutated RpoB that counterbalanced the osmotic stress. Under non-stressed conditions, genes involved in the synthesis and transport of compatible solutes such as glycine-betaine, glutamate or proline were upregulated even without osmotic stimulation, suggesting a "pre-defense" mechanism maybe formed in the rpoB mutant. Under osmotic stressed conditions, genes encoding diverse sugar transporters, which should be down-regulated in the presence of high osmotic pressure, were derepressed in the rpoB mutant. Additional genetic experiments showed that enhancing the expression of the mal regulon, especially for genes that encode the glycoporin LamB and maltose transporter, contributed to the osmotolerance phenotype.

Conclusions: The D654Y single amino acid substitution in RpoB rendered E. coli cells resistant to osmotic stress, probably due to improved cell growth and viability via enhanced sugar uptake under stressed conditions, and activated a potential "pre-defense" mechanism under non-stressed conditions. The findings of this work will be useful for bacterial host improvement to enhance its resistance to osmotic stress and facilitate bio-based organic acids production.
\end{abstract}

Keywords: Osmotolerance, RpoB, Succinic acid, Sugar transporter, RNA-seq

\section{Background}

Escherichia coli has been extensively developed for bio-based production of a wide variety of organic acids, including succinic acid (SA) [1, 2]. Although high yields of SA have been successfully achieved using E. coli as hosts on both laboratory and commercial scales [2-4], cells suffering from osmotic stress during fermentation remains a major barrier for

\footnotetext{
*Correspondence: fan_fy@tib.cas.cn; zhang_xl@tib.cas.cn

${ }^{\dagger}$ Equal contributors

${ }^{1}$ Tianjin Institute of Industrial Biotechnology, Chinese Academy of Sciences,

Tianjin, China

Full list of author information is available at the end of the article
}

hyper SA production. One of the main causes of osmotic stress is a high initial sugar concentration in the medium, which is beneficial for simplifying the carbon source feeding process. However, induced osmotic pressure also negatively impacts robustness and propagating fecundity of the bacterial cells. Alkali is usually added during SA fermentation to maintain the medium at a neutral $\mathrm{pH}[4,5]$. SA accumulates as the dissociated form, disodium succinate, which further aggravate the osmotic stress.

The molecular mechanisms underlying the inhibitory effect due to osmotic stress can be summarized in two 
aspects. First, since sugar molecules cannot freely travel across semi-permeable cell membranes by diffusion, the high concentrations of such external solvents lead to a strong tendency of cytoplasmic water efflux. This dehydration results in shrinkage of the cell volume and malfunction of cell membranes and embedded proteins, leading to osmotic stress [6]. To counterbalance the deleterious effect of osmotic stress, compatible solutes (also called osmoprotectants), such as potassium ions [7], glycine-betaine [8], trehalose [9], glutamate [10], and proline [11] can spontaneously accumulate in cells via de novo synthesis or transport from the medium. Compatible solutes are usually impermeable to the cell membrane, less toxic at high internal concentrations, and not easily catabolized $[6,8]$, which greatly facilitates water remaining within the cytoplasm. In terms of SA production, it was reported that medium supplemented with glycine-betaine or proline improved cell osmotolerance and succinate production in E. coli [12] and Actinobacillus succinogenes [13]. However, it is worth noting that the osmoprotective effects of these compatible solutes are conditional. For example, it was reported that internal glycine-betaine lost its protective effect in the presence of $\mathrm{NaCl}$ concentrations greater than $1 \mathrm{M}$ [14]. Second, inhibition of nutrition uptake might account for the attenuation of cell growth upon external osmolality. Previous studies using an isotopic labeling experiment demonstrated that in the presence of increased osmolality, the activity of nearly all known sugar transport systems in $E$. coli were inhibited, including the glucose phosphotransferase system (PTS), the binding protein mediated maltose transport system, lactose-proton symport system, and melibiose-sodium co-transport system [15]. Sugar transportation defects leading to energy insufficiency could be partially explained by inhibition of DNA replication [16], protein synthesis and respiration [14] under an osmotic stress. It is noteworthy that such inhibitory effects on growth did not lead to cell death because cell growth and metabolic activity were still maintained at a low level [15]. In addition to attenuation of sugar transport, transcriptional repression of genes encoding sugar transporters might also lead to inhibition of sugar uptake. It was experimentally shown that the transcripts abundance of galactitol and maltose transporter genes were drastically downregulated upon $\mathrm{NaCl}$-induced osmotic stress [17], although transcriptional information for other sugar transporters has not been reported.

Our laboratory previously generated an E. coli strain, Suc-T110, for SA production that is highly susceptible to osmotic stress. After maintaining Suc-T110 for more than 1400 generations in a medium containing a high sugar concentration (12\% w/v glucose), an osmotolerant strain, HX024, was obtained. Genome re-sequencing of HX024 showed that only seven genes had non- synonymous point mutations, including $r p o B$ and agaR, which encode transcriptional regulators [4]. In this work, we aimed to discover how these two mutations lead to phenotypic changes in osmotolerance.

\section{Methods}

Strain, medium and growth conditions

Suc-T110, a derivative strain of the E. coli Crooks strain (ATCC\#8739), was used as the parental strain in this study. Genetically modified derivatives of Suc-T110 are listed in Table 1. During strain construction, cultures were grown aerobically in Luria broth (per liter: $10 \mathrm{~g}$ of Difco tryptone, $5 \mathrm{~g}$ of Difco yeast extract, and $10 \mathrm{~g}$ of $\mathrm{NaCl}$ ). For homologous recombination via Red recombinase, which is expressed from a temperature-sensitive plasmid (pKD46) [18], E. coli cultures were grown at $30{ }^{\circ} \mathrm{C}$ to maintain the plasmid. All other cultures were usually grown at $37^{\circ} \mathrm{C}$. Ampicillin $\left(100 \mathrm{mg} \mathrm{L}^{-1}\right)$, kanamycin $\left(50 \mathrm{mg} \mathrm{L}^{-1}\right)$, and chloramphenicol (34 $\left.\mathrm{mg} \mathrm{L}^{-1}\right)$ were added when necessary.

\section{Genetic methods}

Gene knock-outs or overexpression mutants were constructed using a previously described two-step recombination method [18]. Red recombinase was used to facilitate chromosomal gene deletion and modulation [19]. All primers used to construction of mutants are listed in Additional file 1: Table S1. For importation of the mutated $r p o B$ into Suc-T110, a cat-sacB cassette was amplified from plasmid pXZ-CS with the primer set rpoB-QC-cat-up/rpoB-QC-sacB-down, which was used to replace the native $r p o B$ gene in the Suc-T110 chromosome via homologous recombination. Then the mutated rpoB gene was amplified from strain HX024 with primer set $r p o B$-up/rpoB-down, which was used to replace the cat-sacB cassette via a second recombination event.

Table 1 Strains used in this study

\begin{tabular}{|c|c|c|}
\hline Strains & Genotype & Source \\
\hline Suc-T110 & $\begin{array}{l}\text { E.coli ATCC\#8739, } \Delta l d h A, \\
\Delta p f l B, \triangle p t s l, P p c k^{*} \text {-galP, } \\
\text { Ppck*-pck }\end{array}$ & [41] \\
\hline HX024 & $\begin{array}{l}\text { Suc-T110, } \triangle a c k A-p t a, \\
\text { Ppck*-aceBA, Ppck*-dcuC, } \\
\triangle m g s A, \text { adaptively evolved } \\
\text { for } 1440 \text { generations }\end{array}$ & {$[4]$} \\
\hline RpoBD645Y & Suc-T110:: rpoB (D654Y) & In this study \\
\hline AgaRR109W & Suc-T110:: agaR (R109W) & In this study \\
\hline OV-LamB & $\begin{array}{l}\text { Suc-T110, Ppck*- } \\
\text { malk-lamB-malM }\end{array}$ & In this study \\
\hline RpoBD645Y/AmalEFG & $\begin{array}{l}\text { RpoBD645Y, } \\
\text { AmalE-malF-malG }\end{array}$ & In this study \\
\hline 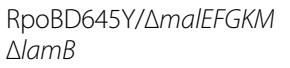 & $\begin{array}{l}\text { RpoBD645Y, } \Delta \text { male-malF-malG } \\
\text { smalK-lamB-malM }\end{array}$ & In this study \\
\hline
\end{tabular}

Ppck* stand for a mutant of the E. coli pck promoter which with a G-to-A mutation at position -64 relative to the ATG start codon 
Since cells containing the $s a c B$ gene die when grown on sucrose due to the accumulation of levan, transformants containing the mutated $r p o B$ gene were selected for resistance to sucrose [18]. The mutated agaR gene was cloned into Suc-T110 by a similar method, and a single deletion of the malEFG operon as well as a double deletion of the malEFG and malK-lamB-malM operons in Suc-T110 were generated using the the same method. For over-expression of $\operatorname{lamB}$ in Suc-T110, the promoter for the malK-lamB-malM operon was replaced with a constitutive strong promoter $P p c K^{*}$ using the two-step recombination method as mentioned above.

\section{Growth under normal or osmotic conditions}

Fresh colonies were inoculated into $250-\mathrm{mL}$ flasks containing $30 \mathrm{~mL}$ of modified NBS mineral salts medium [20] [per liter: $3.5 \mathrm{~g}$ of $\mathrm{KH}_{2} \mathrm{PO}_{4}, 5.0 \mathrm{~g}$ of $\mathrm{K}_{2} \mathrm{HPO}_{4}$, $3.5 \mathrm{~g}$ of $\left(\mathrm{NH}_{4}\right)_{2} \mathrm{HPO}_{4}, 0.25 \mathrm{~g}$ of $\mathrm{MgSO}_{4} \cdot 7 \quad \mathrm{H}_{2} \mathrm{O}$, $15 \mathrm{mg} \mathrm{CaCl}_{2} \cdot 2 \mathrm{H}_{2} \mathrm{O}, 0.5 \mathrm{mg}$ of thiamine, $10.0 \mathrm{~g}$ of $\mathrm{KHCO}_{3}$ and $0.15 \mathrm{~g}$ of betaine $\mathrm{HCl}$ and $1 \mathrm{ml}$ of trace metal stock]. The trace metal stock was prepared in $0.1 \mathrm{M} \mathrm{HCl}$ and contained the following (per liter):1.6 g of $\mathrm{FeCl}_{3}, 0.2 \mathrm{~g}$ of $\mathrm{CoCl}_{2} \cdot 6 \mathrm{H}_{2} \mathrm{O}, 0.1 \mathrm{~g}$ of $\mathrm{CuCl}_{2}, 0.2 \mathrm{~g}$ of $\mathrm{ZnCl}_{2} \cdot 4 \mathrm{H}_{2} \mathrm{O}, 0.2 \mathrm{~g}$ of $\mathrm{NaMoO}_{4}, 0.05 \mathrm{~g}$ of $\mathrm{H}_{3} \mathrm{BO}_{3}$. The medium contained $20 \mathrm{gL}^{-1}$ glucose, and cultures were grown at $37{ }^{\circ} \mathrm{C}$ and $250 \mathrm{rpm}$ for $12 \mathrm{~h}$. Cultures were then transferred to 500-mL fermentation vessels, which contained $250 \mathrm{~mL}$ of NBS mineral salts medium supplemented with $50 \mathrm{gL}^{-1}$ or $120 \mathrm{gL}^{-1}$ glucose to represent the non-stressed or osmotic stressed conditions, respectively. Then, the cultures were incubated anaerobically for $96 \mathrm{~h}$ at $37{ }^{\circ} \mathrm{C}$ and $150 \mathrm{rpm}$. Cell mass was estimated by measuring the optical density at $550 \mathrm{~nm}\left(\mathrm{OD}_{550}\right)$ as described previously [9]. The succinate and glucose concentration in the medium were measured by high-performance liquid chromatography according to a previously reported protocol [21].

\section{RNA extraction}

After $48 \mathrm{~h}$ of fermentation under normal $(5 \% \mathrm{w} / \mathrm{v}$ glucose) or osmotic-stressed ( $12 \% \mathrm{w} / \mathrm{v}$ glucose) conditions, samples of Suc-T110 and RpoBD645Y were harvested for total RNA isolation. Extraction and additional oncolumn DNase I treatment was performed with the RNeasy mini kit (Qiagen) according to the manufacturer's protocol. The purified RNA was assessed with an Agilent 2100 Bioanalyser (Agilent) and quantified using a NanoDrop ND-1000 spectrophotometer (NanoDrop Technologies).

\section{RNA sequencing and data analysis}

Four 90-nt paired-end RNA-seq libraries were generated at Beijing Genomics Institute (BGI, Shenzhen, China) with the HiSeq 2000 platform. Quality control of sequencing reads was performed with the NGS QC Toolkit (version 2.3.3) [22], and the obtained high-quality reads were aligned to the $E$. coli ATCC\#8739 genome (GenBank CP000946.1) with Bowtie 2 (version 2.2.5) [23]. The aligned reads stored in SAM format file and the raw counts for reads mapping to unique gene were then tallied with HTSeq-count scripts (0.6.0) with the intersection-nonempty resolution mode [24]. Abundance for each transcript was calculated using the Reads Per Kilobase per Million (RPKM) measure as described previously [25]. Differential expression gene calling was performed with R package NOISeq (version 2.6.0) [26] with "probability of differential expression (q value)" $\geq 0.9$ and $\mid \log 2$ Ratio $\mid \geq 1$ as the final cut-off. MIPS FunCat online tools [27] were used to annotate genes with altered expression, and enriched categories $(P$ value $<0.001)$ were marked out.

\section{Statistical significance tests}

Unless otherwise noted, all experiments were performed in triplicate, and statistical tests for significance were determined via a one-way ANOVA using R (version 3.1.1).

\section{Results and Discussion}

A point mutation in $r p o B$ confers resistance to osmotic stress

As stated above, the osmotolerant mutant HX024 had single, non-synonymous point mutations in both $r p o B$ (DNA sequence change, G1960T; Protein sequence change, D654Y) and agaR (DNA sequence change, C325T; Protein sequence change, R109W). Given that the physiological functions of the two encoded proteins are both associated with transcriptional regulation, we speculated that the two mutations are likely to cause phenotypic changes in osmotolerance. To test our hypothesis, the coding regions of the mutated $r p o B$ and $a g a R$ were separately amplified from HX024 and were used to replace the corresponding non-mutated genes in Suc-T110. The obtained mutation strains were designated as RpoBD645Y [Suc-T110::rpoB (D654Y)] and AgaRR109W [Suc-T110::agaR (R109W)]. When cultured in $5 \% \mathrm{w} / \mathrm{v}$ glucose, no obvious growth difference was detected for either RpoBD645Y or AgaRR109W in comparison to Suc-T110 (Fig. 1a). However, under high osmolarity conditions $(12 \% \mathrm{w} / \mathrm{v}$ glucose $)$, the growth of both AgaRR109W and Suc-T110 significantly decreased to approximately $45 \%$ of that under normal conditions at $96 \mathrm{~h}$, whereas RpoBD645Y showed normal growth under these conditions, i.e., similar to growth in $5 \% \mathrm{w} / \mathrm{v}$ glucose (Fig. 1a). In addition, under high osmolarity conditions, only RpoBD645Y showed a similar SA production titer after $96 \mathrm{~h}$ of fermentation as normal growth condition, whereas the other two strains showed 

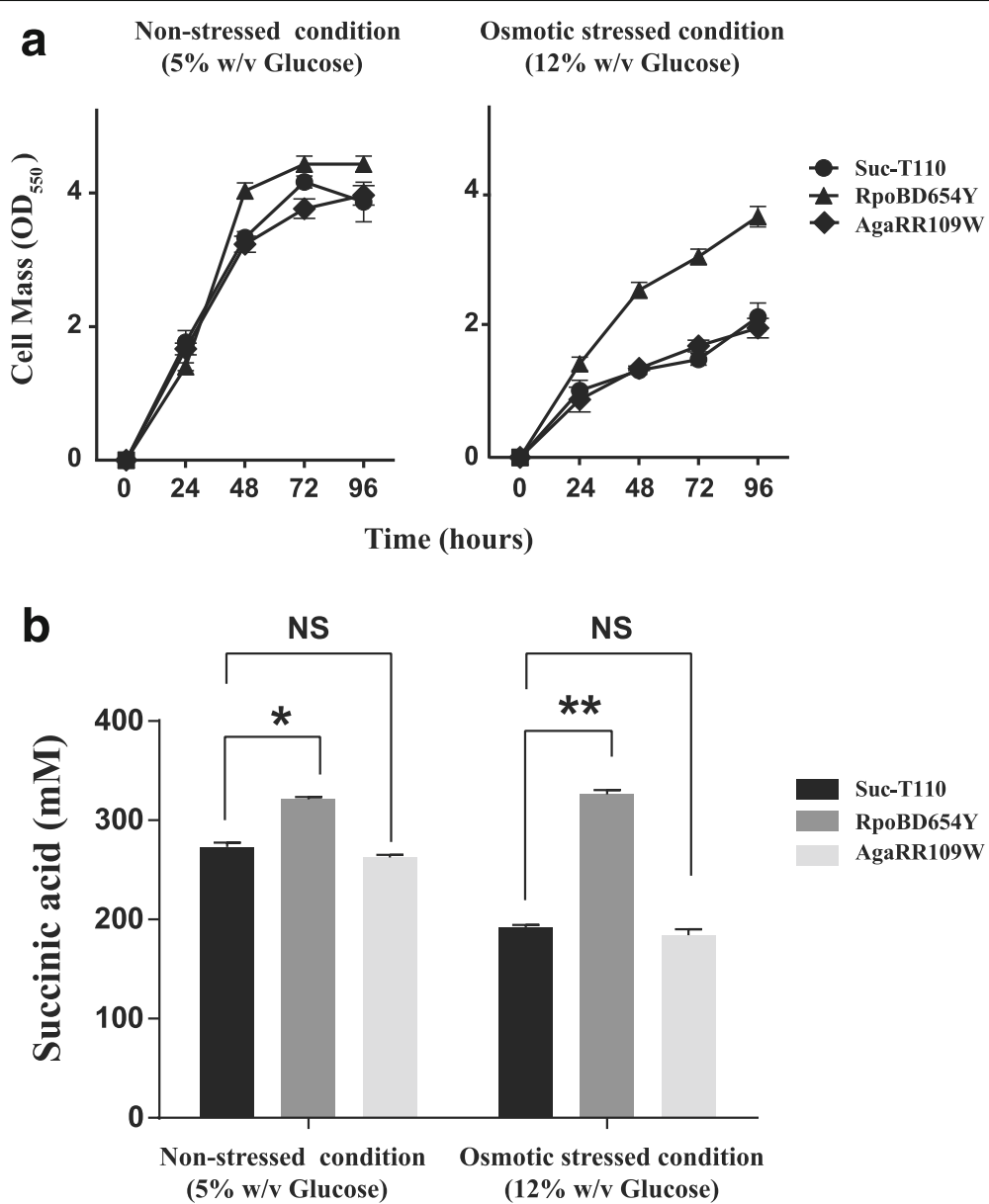

Fig. 1 A point mutation in $r p o B$ conferred improved cell growth and succinic acid production to Suc-T110 under osmotic stress. a Cell growth of RpoBD645Y (A Suc-T110 derivative harboring rpoB ${ }^{G 1960 T}\left[R_{p o B}{ }^{D 654 Y}\right]$ ), and AgaRR109W (A Suc-T1 10 derivative harboring agaR ${ }^{C 325 T}\left[\right.$ AgaR $\left.^{R 109 W}\right]$ ) along with the parental strain, Suc-T110, under normal ( $5 \% \mathrm{w} / \mathrm{v}$ glucose) or osmotic stress ( $12 \% \mathrm{w} / \mathrm{v}$ glucose) conditions. b SA production by RpoBD645Y, AgaRR109W, and Suc-T110 after $96 \mathrm{~h}$ of fermentation. Data are the mean with the standard error of the mean (SEM, $n=3$ ). The significance of differences was calculated by one-way ANOVA; asterisks indicate a significant difference from the control $\left(^{* *} P<0.01\right.$; $P<0.05$; NS = not significant)

lower production (Fig. 1b). These results suggested that the mutated $r р о B$ but not the mutated agaR rendered Suc-T110 osmotolerant.

In E. coli ATCC\#8739, the gene rpoB (EcolC_4038) encodes the $\beta$-subunit of DNA-dependent RNA polymerase (RNAp). RNAp, which is the primary enzyme responsible for gene transcription in prokaryotes, consists of five subunits $\left(\alpha 2 \beta \beta^{\prime} \omega\right)$ and is associated with one of several alternative sigma $(\sigma)$ factors. Sigma factors, such as $\sigma^{\mathrm{S}}$ in E. coli [28], $\sigma^{\mathrm{B}}$ in Listeria monocytogenes [29], and RpoN in Campylobacter jejuni [30], have been reported to be involved in the regulation of osmotic stress responses. However, direct evidence for the involvement of the RNAp $\beta$-subunit in osmotolerance has not yet been reported. An analysis of the protein domain architecture of the RNAp $\beta$-subunit using SMART online tools [31] showed that the D654Y mutation was located in a Pfam domain (PF10385) called the external one region of the polymerase. However, the function of this domain has not been characterized. Given that the $\beta$-subunit, along with the $\beta$ ' subunits, constitute the active center of RNAp [32], we speculated that the D654Y mutation might lead to a conformational change in the RNAp active site, and therefore, affect the overall gene transcription pattern. It has been shown that point mutations in the $\beta$ subunit of Enterococci RNAp conferred the capability of adaption to drug stress induced by cephalosporin [33], which probably occurs via a similar mechanism.

Transcriptomic profiling revealed mutated RpoB caused osmotic response genes upregulated under non-osmotic stressed conditions

To decipher how the mutated RpoB orchestrated global gene transcription to counteract osmotic stress, RNAseq analyses were performed on the parental strain 
Suc-T110 and osmotolerant strain RpoBD645Y under both normal (5\% w/v glucose) and high osmolality $(12 \% \mathrm{w} / \mathrm{v}$ glucose) conditions (Additional file 2: Table S2, Fig. 2a).

Of the 4200 annotated genes, 128 genes showed altered expression in the strain containing the RpoB D654Y mutation under normal osmolality; with 38 upregulated and 90 downregulated genes. Functional enrichment of the 38 up-regulated genes by MIPS FunCat tools showed that 11 genes were engaged in biological functions of amino acid metabolism ( $P$-value $=4.60 \mathrm{E}-05 ;$ Additional file 3: Table S3, P1 and P3, Fig. 2b). As mentioned above, bacterial cells commonly accumulate glutamate and proline to high levels under osmotic stress. The transcriptional abundance of genes involved in the metabolism of glutamate and aspartate (which can be converted into glutamate through transamination [34]), such as EcolC_2869, EcolC_3652, EcolC_3653, EcolC_1833, EcolC_4075, and EcolC_3651, were markedly increased in RpoBD645Y under normal
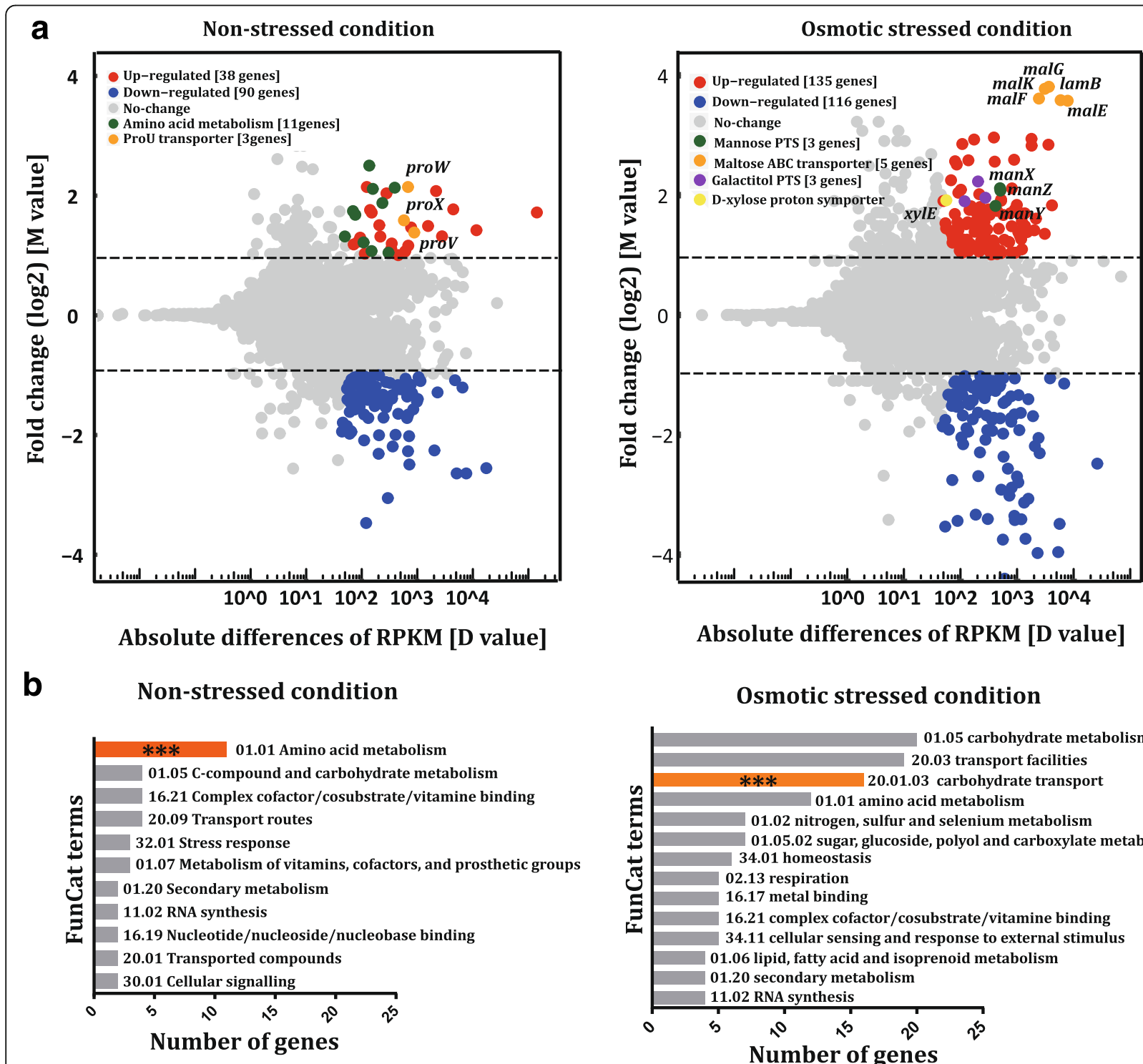

Absolute differences of RPKM [D value]

\section{Osmotic stressed condition}

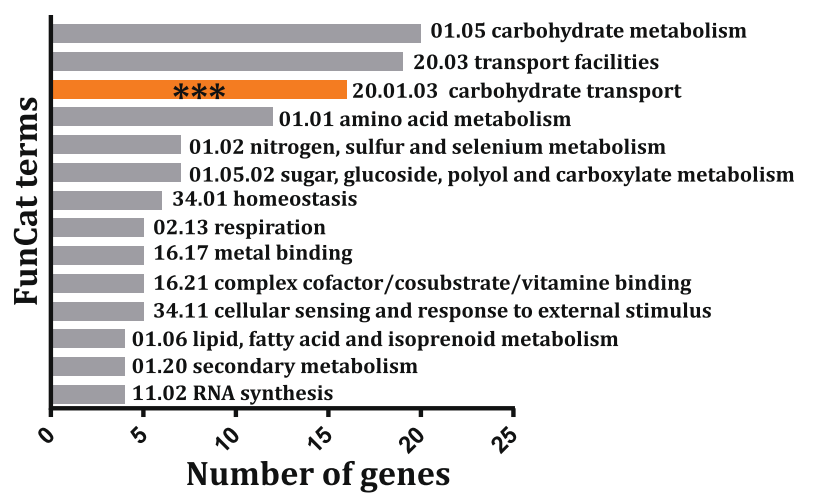

Fig. 2 Transcriptional response to osmotic stress in Suc-T110 and RpoBD645Y cells. a The altered gene expression patterns elicited by the rpoB mutation under either normal (left panel) or osmotic stress (right panel) conditions were separately plotted in as an MD-plot. M values ( $Y$ axis) represent the log2 normalized fold changes. $D$ values ( $X$ axis) are the absolute differences in RPKM between Suc-T110 and RpoBD645Y. Each point represents a transcript with a detected expression level. Red or blue points represent differentially expressed genes with increased or decreased abundance, respectively ( $q$ value $\geq 0.90$ and $\mid \log 2$ ratio $\geq 1$ ), whereas gray points are genes with no differences in expression. Transcripts used to interpret the osmotolerance phenotype are plotted in different colors. b Functional enrichment analysis of the upregulated genes. Typical FunCat terms are listed, and the asterisks $\left(^{* * *}\right)$ indicate a significant enrichment (unadjusted $P$-value $<0.001$ ) 
osmolality. Genes involved in proline metabolism were not detected in the up-regulated gene set. However, the expression of genes encoding the major proline transporter system ProU $[6,7]$ was markedly enhanced. Transcription levels of genes located in the proU operon, including proX (EcolC_1027, encoding substrate-binding protein), proW (EcolC_1028, encoding permease), and proV (EcolC_1029, encoding ATP-binding protein), increased 3- to 4- fold in RpoBD645Y. These results were consistent with previous observations that proline accumulation in E. coli was due to enhanced transportation and not by synthesis $[6,35]$. In addition, the ProU system also functions as the major system for glycine betaine uptake in E. coli [36]. A previous study showed that ProU-mediated glycine betaine transport was osmotically stimulated at the level of gene expression [6]. However, to our knowledge, the observation that the proU operon highly expressed under non-osmotic stress conditions has not yet been reported. Given that our modified NBS mineral salts medium contains a small amount of betaine $(1 \mathrm{mM})$, we speculated that this compatible solute could be taken up into RpoBD645Y via ProU under non-osmotic stressed conditions, although further study is needed to obtain more direct evidence. In summary, the $r p o B$ mutation probably conferred $E$. coli with the ability to mount a "predefense" mechanism, such that the osmotic response genes were activated under non-osmotic stressed conditions, which helped the cells better adapt to subsequent osmotic shock.

\section{Transcriptomic profiling showed that mutated RpoB conferred osmotolerance via derepression of sugar transporters}

Under a high osmolality, 244 differentially transcribed genes were identified in RpoBD645Y when compared with Suc-T110; 132 were upregulated and 112 were downregulated (Additional file 3: Table S3, P2). Since genes involved in glutamate synthesis and proline/glycine betaine transportation showed similar transcription levels in RpoBD645Y and Suc-T110 (Additional file 2: Table S2 and Additional file 3: Table S3), strategies other than "pre-defense" mechanisms might be adopted by RpoBD645Y to survive under high osmolality. Functional analysis of 132 upregulated genes indicated that the most significantly enriched physiological function of RpoBD645Y upon osmotic stress was associated with carbon source transportation (FunCat term: 20.01.03 Ccompound and carbohydrate transport, $P$-value $=9.51 \mathrm{E}$ 05; Additional file 3: Table S3, P5, Fig. 2b). This group included genes encoding diverse sugar transporters. The expression levels of these genes have been reported to be drastically reduced under osmotic stress conditions $[17,37,38]$. We found that these sugar transporter genes were drastically repressed in Suc-T110 under osmotic stress, whereas in RpoBD645Y, their expression maintained stable under osmotic stress. For example, transcript levels of malE (EcolC_3995), malF (EcolC_3996), malG (EcolC_3997) malK (EcolC_3994), and lamB (EcolC_3993), which encode individual subunits of the maltose ABC (ATP-binding cassette) transporter, decreased 10- to 14- fold in Suc-T110 compared to the corresponding levels in RpoBD645Y under osmotic stress. Meanwhile, genes encoding galactose PTS (EcolC_1645, EcolC_1646, and EcolC_1647), mannose PTS (ManY: EcolC_1814 and ManZ: EcolC_1813), Dxylose proton symporter XylE (EcolC_3998), and the melibiose-sodium co-transport system (EcolC_3907) were decreased 2- to 5- fold in Suc-T110 when compared to the corresponding levels in RpoBD645Y. These data demonstrated that repression effects on sugar transport genes were alleviated in RpoBD645Y due to the $r p o B$ mutation. Based on FunCat analysis, no other processes with potential osmotic responsive functions were markedly enriched (cut-off, $P$ value $\leq$ 0.001) in RpoBD645Y (Additional file 3: Table S3, P5, Fig. 2b). We assumed that maintenance of sugar transporter gene expression may be helpful for maintaining growth under osmotic stress, which might be the primary reason for the osmotic stress resistance of RpoBD645Y.

\section{The porin LamB contributes to osmotolerance in RpoBD645Y}

In $E$. coli, the process of glucose uptake can be divided into two steps. Glucose is first internalized into the periplasm via porins located in the outer membrane, and then imported into the cytoplasm through diverse inner membrane PTS and non-PTS sugar transporters. Three porins including $\mathrm{OmpF}, \mathrm{OmpC}$, and $\mathrm{LamB}$, have been reported to be involved in glucose internalization into the periplasm [37]. In addition, the expression of ompF and ompC in $E$. coli has been shown to be regulated by osmotic stimuli. OmpC predominates at high osmolarity, while OmpF expression is repressed [39], which is consistent with our RNA-seq data (Additional file 2: Table S2). Previous work also demonstrated that OmpC and OmpF were required for cell growth under hyperosmosis at an alkaline $\mathrm{pH}$ and hypo-osmotic stress at an acidic $\mathrm{pH}$; however, they were not required for growth at near neutral $\mathrm{pH}$ under both hyper- and hypo-osmosis [39]. These conclusions suggest that $\mathrm{OmpC}$ and $\mathrm{OmpF}$ might not contribute to osmotolerance of RpoBD645Y because fermentations were performed at a neutral $\mathrm{pH}$. This raised the possibility that osmotolerance may result from the elevated expression of lamb. Therefore, we then overexpressed $\operatorname{lamB}$ in strain Suc-T110. In E. coli, malK (encoding ATP-binding component of the maltose 
$\mathrm{ABC}$ transporter), lam $B$, and malM (encoding periplasmic protein with unclear function) are located in the same operon. For the overexpression experiment, the native promoter of the malK-lamB-malM operon was changed to a strong constitutive promoter $P p c k^{*}$ [20], via homologous recombination to obtain the lamb-overexpression mutant OV-lamB. Under osmotic stress, we found that the growth and succinate production of this strain were increased $47 \%$ and $42 \%$, respectively compared to the corresponding values in Suc-T110 (Fig. 3a and b). In addition, OV-lamB had an average glucose consumption rate at $0.5 \mathrm{~g} \mathrm{~L}^{-1} \mathrm{~h}^{-1}$ during a 96-h fermentation, which was $25 \%$ higher than that of Suc-T110 (Fig. 3c). These results suggest that LamB contributed to the osmotolerance of HX024. Previous work demonstrated that LamB contribute about $70 \%$ of the total glucose import capacity of the cell under glucose limited conditions [40], suggesting that LamB had a high affinity for glucose under certain conditions. Thus, the contribution of $\operatorname{lamB}$ overexpression to osmotolerance could be due to enhanced glucose uptake capability under osmotic stress.
Derepression of the inner membrane-associated maltose $A B C$ transporter confers osmotolerance

In addition to porin proteins, a number of sugar transporters located on the inner membrane were also derepressed in RpoBD645Y under high osmolarity. Thus, additional experiments were carried out to test whether modifying the expression of these transporter genes would alter osmotolerance.

It should be noted that in Suc-T110, the galP gene, which encodes galactose permease, was genetically modified by replacing its native promoter with a strong constitutive promoter Ppck* [41]. In E. coli, glucose uptake occurs mainly through the glucose phosphoenolpyruvate: carbohydrate phosphotransferase system (glucose PTS) [42]. However, this internalization process consumes half of the intracellular phosphoenolpyruvate (PEP) for use in glucose phosphorylation, which results in an insufficient PEP supply for SA production [20, 43]. For this reason, the PTS system was impaired in SucT110 by deletion of ptsI (encoding PTS enzyme I), and GalP was constitutively expressed to restore glucose
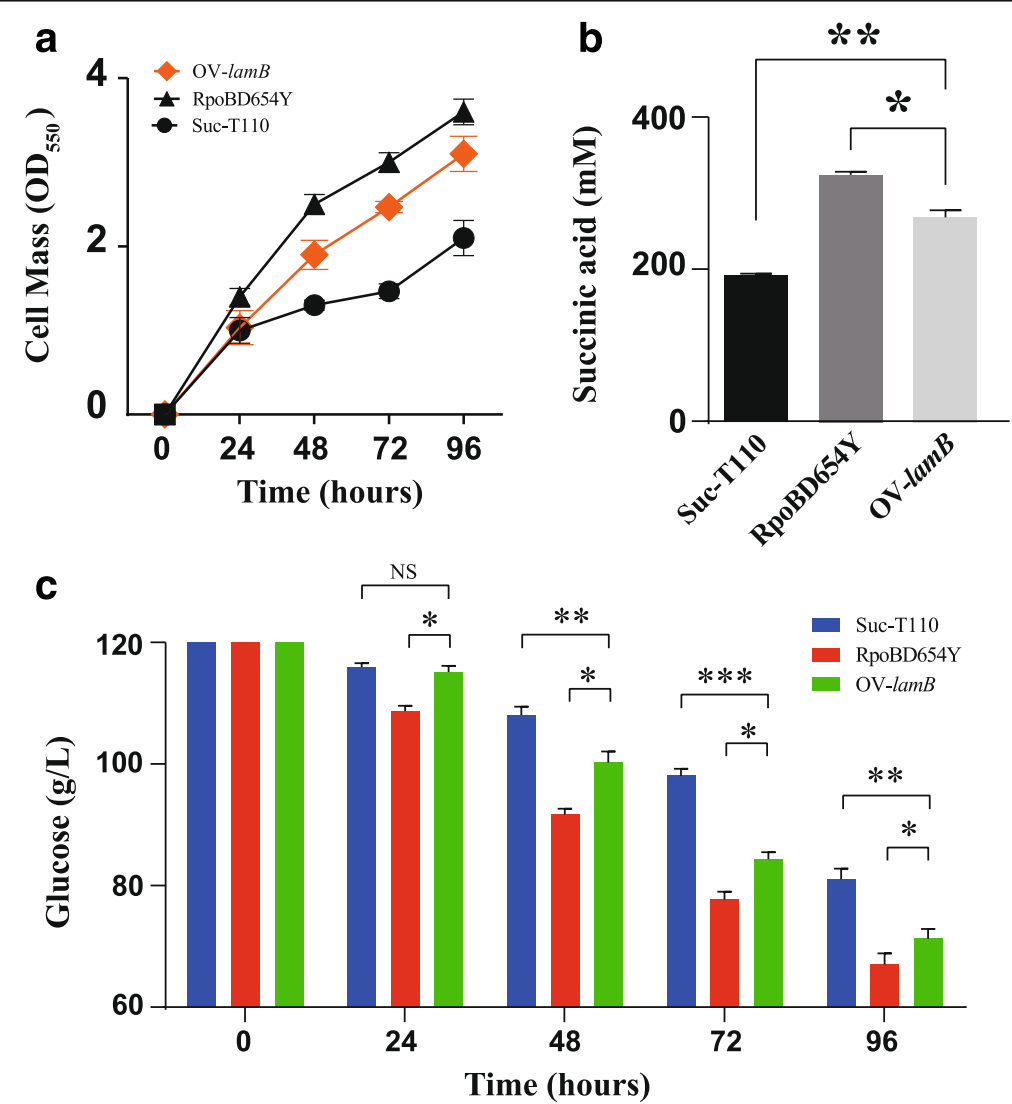

Fig. 3 lamB overexpression rescued cell growth and the succinate production defect of Suc-T110 under osmotic stress. a Growth of lamBoverexpressing Suc-T110 (OV-lamB) under osmotic stress (12\% w/V glucose). b SA production of the OV-lamB strain after $96 \mathrm{~h}$ of fermentation. c Glucose consumption by OV-lamB. Strains RpoBD645Y and Suc-T110 were used as controls. Data are the mean with the standard error of the mean (SEM, $n=3$ ). The significance of differences was calculated with one-way ANOVA; the asterisks indicate a significant difference from the controls $\left({ }^{* *} P<0.001 ;{ }^{* *} P<0.01 ;{ }^{*} P<0.05 ; \mathrm{NS}=\right.$ not significant $)$ 
transport and utilization [38, 44]. Although relatively high galP expression was maintained in Suc-T110 even under osmotic stress, cell growth was markedly inhibited (Fig. 1a), suggesting that GalP could not effectively transport glucose under high osmolality. We speculated that this phenotype may be caused by ineffective internalization of glucose into the periplasm. In Suc-T110, LamB and OmpF were repressed, while OmpC was induced under osmotic stress, suggesting that glucose was transported into the periplasm mainly via the OmpC porin. However, OmpC has a smaller pore diameter than other porins [45], suggesting that glucose probably diffused into the periplasm at a lower rate, which would limit the function of GalP. One of the derepressed sugar transporters, D-xylose-proton symporter XylE could not transport glucose [46], and the galactitol and the mannose PTS system became non-functional in Suc-T110 due to the ptsI deletion. Thus, only GalP and maltose transporter might contribute to the osmotolerance of RpoBD645Y by enhancing glucose uptake. In E. coli, the maltose regulon consists of ten genes that are located on five operons [47]. To obtain genetic evidence that the maltose $\mathrm{ABC}$ transporter contributed to the osmotolerance of RpoBD645Y, the malEFG operon, which encodes the core subunits of the maltose transporter, was deleted in RpoBD645Y. Cell growth and succinate production of the resulting strain, RpoBD645Y/AmalEFG, decreased by $24 \%$ (Fig. $4 \mathrm{a}$ ) and 20\%, respectively (Fig. $4 \mathrm{~b}$ ), compared with that of RpoBD645Y after $96 \mathrm{~h}$ of fermentation. The average glucose consumption rate of RpoBD645Y/DmalEFG was $0.48 \mathrm{~g} \mathrm{~L}^{-1} \mathrm{~h}^{-1}$, which was approximately $20 \%$ lower than that of Suc-T110 (Fig. 4c). Since LamB usually functions synergistically with the maltose $A B C$ transporter, the adjacent operons of malEFG and malK-

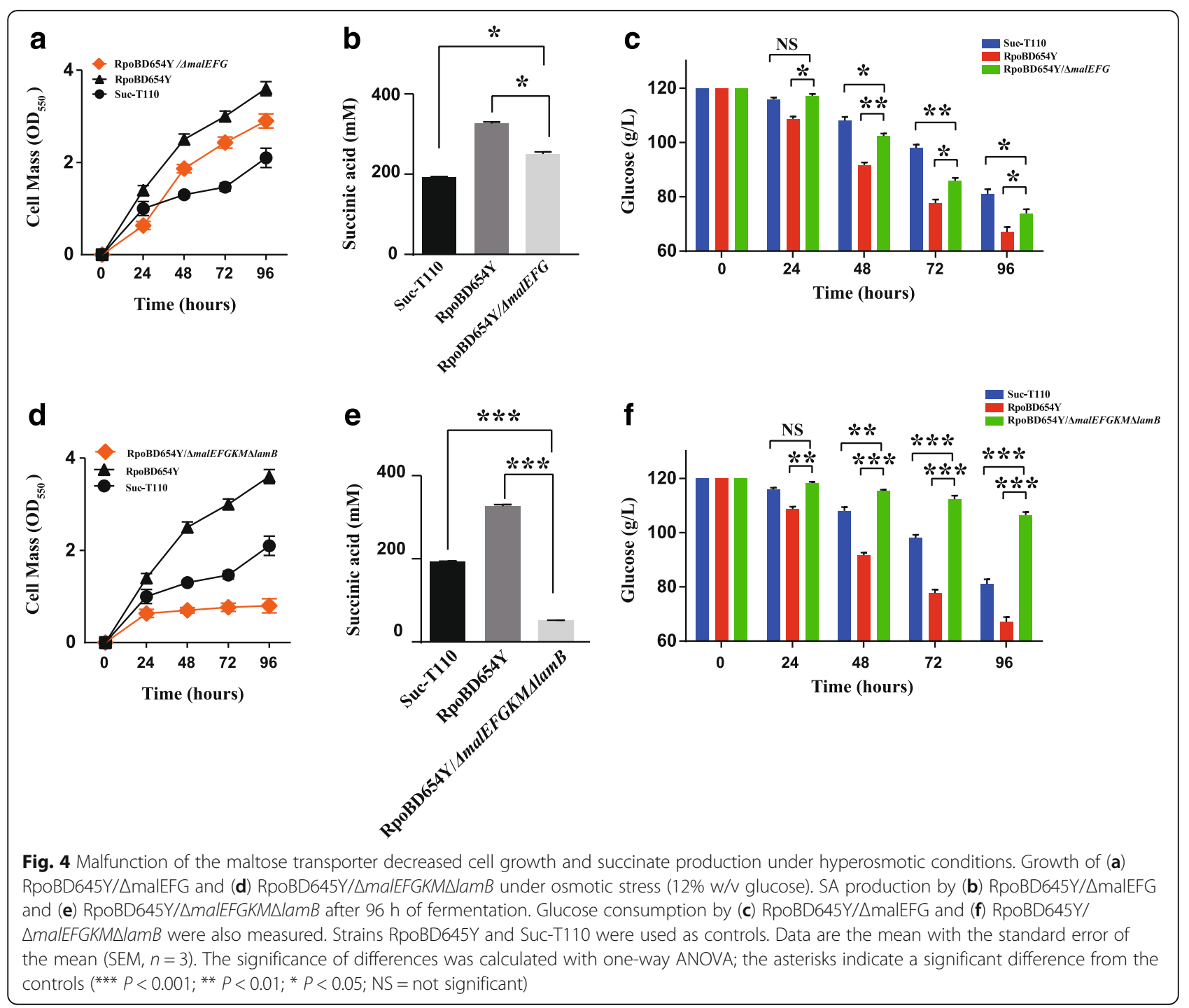




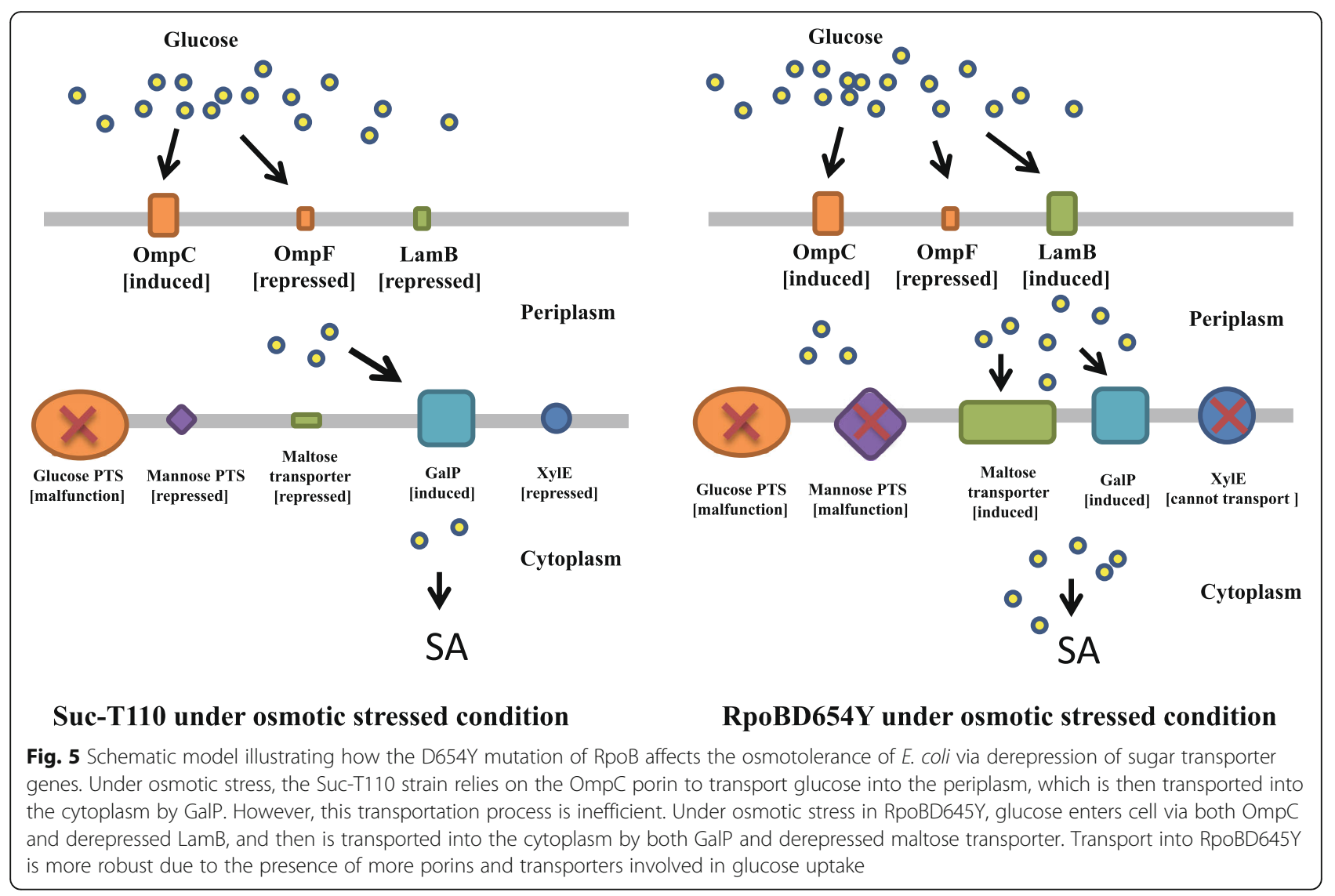

lamB-malM were deleted in RpoBD645Y. Cell growth and succinate production of this double deletion mutant (RpoBD645Y/AmalEFGKM $\triangle$ lamB) were significantly lower than those of RpoBD645Y, which was even worse than Suc-T110 (Fig. 4d, e). The average glucose consumption rate of RpoBD645Y/DmalEFGKM $\triangle$ lamB was only $0.14 \mathrm{~g} \mathrm{~L}^{-1} \mathrm{~h}^{-1}$, which corresponded to $34 \%$ and $25 \%$ of the rates of Suc-T110 and RpoBD645Y, respectively (Fig. 4f). This suggested that derepression of the mal regulon was involved in the osmotolerance of RpoBD645Y (Fig. 5).

\section{Conclusion}

A novel point mutation (D654Y) within RpoB was identified in this work, which improved the osmotolerance of $E$. coli. This mutation affected the transcriptional activity of RpoB, leading to upregulation of several osmotic response genes which involved in the biosynthesis or transportation of compatible solutes under non-osmotic stressed conditions, probably contributes to compatible solute accumulation. This mutation also enhanced glucose uptake under high sugar osmolality via derepression of the mal regulon. Thus, this mutation can be used to improve cell growth under osmotic stress and increase the production of succinate and other organic acids.

\section{Additional files}

Additional file 1: Table S1. Primer list. (DOCX $18 \mathrm{~kb}$ )

Additional file 2: Table S2. RNA-seq read profiles mapped to the genome of E.coli ATCC\#8739. (XLSX 532 kb)

Additional file 3: Table S3. Differential expression analysis and functional groups of genes with altered expression. (XLSX $953 \mathrm{~kb}$ )

\section{Abbreviations}

ATP: Adenosine triphosphate; EMP: Embden-Meyerhof-Parnas (EMP) glycolytic pathway; G6P: Glucose-6-phosphate; PEP: Phosphoenolpyruvate; PTS: Phosphoenolpyruvate, carbohydrate phosphotransferase system; RNAp: DNA-dependent RNA polymerase; RPKM: Reads Per Kilobase per Million; rTCA: Reductive tricarboxylic acid cycle; SA: Succinic acid

Acknowledgements

The authors wish to thank Ms. Ying Qin for the help in the experiments.

\section{Funding}

This research was supported by grants from the Key deployment project of the Chinese Academy of Sciences (ZDRW-ZS-2016-3), National High Technology Research and Development Program of China (2014AA021205), National Natural Science Foundation of China (31522002 and 31300089), and Tianjin Key Technology R\&D program of Tianjin Municipal Science and Technology Commission (13ZCZDSY05300).

Availability of data and materials

The RNA-seq data discussed in this publication have been deposited in NCBI's Gene Expression Omnibus [48] and are accessible through GEO Series accession number GSE84769 (https://www.ncbin.nlm.nih.gov/geo/query/ acc.cgi?acc=GSE84769). 


\section{Authors' contributions}

$M X, X i Z, F F$, and $X u Z$ designed the study and advised on protocols. MX and $X i Z$ carried out the experimental procedures. $H X, J T, R L$, and $C B$ helped with experimental procedures and manuscript preparation. The manuscript was read and approved by all the authors.

\section{Competing interests}

The authors declare that they have no competing interests.

\section{Consent for publication}

Not applicable.

\section{Ethics approval and consent to participate}

Not applicable.

\section{Author details}

${ }^{1}$ Tianjin Institute of Industrial Biotechnology, Chinese Academy of Sciences, Tianjin, China. ${ }^{2}$ Key Laboratory of Systems Microbial Biotechnology, Chinese Academy of Sciences, 32 West 7th Ave, Tianjin Airport Economic Park, Tianjin 300308, China. ${ }^{3}$ University of Chinese Academy of Sciences, Beijing, China.

\section{Received: 11 August 2016 Accepted: 10 February 2017}

\section{Published online: 13 February 2017}

\section{References}

1. Jansen ML, van Gulik WM. Towards large scale fermentative production of succinic acid. Curr Opin Biotechnol. 2014;30:190-7.

2. Ahn JH, Jang YS, Lee SY. Production of succinic acid by metabolically engineered microorganisms. Curr Opin Biotechnol. 2016;42:54-66.

3. Chen C, Ding S, Wang D, Li Z, Ye Q. Simultaneous saccharification and fermentation of cassava to succinic acid by Escherichia coli NZN111. Bioresour Technol. 2014;163:100-5.

4. Zhu X, Tan Z, Xu H, Chen J, Tang J, Zhang X. Metabolic evolution of two reducing equivalent-conserving pathways for high-yield succinate production in Escherichia coli. Metab Eng. 2014;24:87-96.

5. Chen J, Zhu X, Tan Z, Xu H, Tang J, Xiao D, Zhang X. Activating C4dicarboxylate transporters DcuB and DcuC for improving succinate production. Appl Microbiol Biotechnol. 2014;98(5):2197-205.

6. Csonka LN. Physiological and genetic responses of bacteria to osmotic stress. Microbiol Rev. 1989;53(1):121-47.

7. Gowrishankar J. Identification of osmoresponsive genes in Escherichia coli: evidence for participation of potassium and proline transport systems in osmoregulation. J Bacteriol. 1985;164(1):434-45.

8. Perroud B, Le Rudulier D. Glycine betaine transport in Escherichia coli: osmotic modulation. J Bacteriol. 1985;161(1):393-401.

9. Miller EN, Ingram LO. Combined effect of betaine and trehalose on osmotic tolerance of Escherichia coli in mineral salts medium. Biotechnol Lett. 2007; 29(2):213-7.

10. Richey B, Cayley DS, Mossing MC, Kolka C, Anderson CF, Farrar TC, Record MT. Variability of the intracellular ionic environment of Escherichia coli. Differences between in vitro and in vivo effects of ion concentrations on protein-DNA interactions and gene expression. J Biol Chem. 1987;262(15):7157-64.

11. Britten RJ, Mc CF. The amino acid pool in Escherichia coli. Bacteriol Rev. 1962:26:292-335

12. Andersson C, Helmerius J, Hodge D, Berglund KA, Rova U. Inhibition of succinic acid production in metabolically engineered Escherichia coli by neutralizing agent, organic acids, and osmolarity. Biotechnol Prog. 2009; 25(1):116-23.

13. Fang X, Li J, Zheng X, Xi Y, Chen K, Wei P, Ouyang PK, Jiang M. Influence of osmotic stress on fermentative production of succinic acid by Actinobacillus succinogenes. Appl Biochem Biotechnol. 2011;165(1):138-47.

14. Houssin C, Eynard N, Shechter E, Ghazi A. Effect of osmotic pressure on membrane energy-linked functions in Escherichia coli. Biochim Biophys Acta. 1991;1056(1):76-84.

15. Roth WG, Leckie MP, Dietzler DN. Osmotic stress drastically inhibits active transport of carbohydrates by Escherichia coli. Biochem Biophys Res Commun. 1985;126(1):434-41.

16. Meury J. Glycine betaine reverses the effects of osmotic stress on DNA replication and cellular division in Escherichia coli. Arch Microbiol. 1988; 149(3):232-9.
17. Cheung KJ, Badarinarayana V, Selinger DW, Janse D, Church GM. A microarray-based antibiotic screen identifies a regulatory role for supercoiling in the osmotic stress response of Escherichia coli. Genome Res. 2003;13(2):206-15.

18. Jantama K, Haupt MJ, Svoronos SA, Zhang X, Moore JC, Shanmugam KT, Ingram LO. Combining metabolic engineering and metabolic evolution to develop nonrecombinant strains of Escherichia coli $\mathrm{C}$ that produce succinate and malate. Biotechnol Bioeng. 2008;99(5):1140-53.

19. Datsenko KA, Wanner BL. One-step inactivation of chromosomal genes in Escherichia coli K-12 using PCR products. Proc Natl Acad Sci U S A. 2000; 97(12):6640-5

20. Zhang $X$, Jantama K, Moore JC, Jarboe LR, Shanmugam KT, Ingram LO Metabolic evolution of energy-conserving pathways for succinate production in Escherichia coli. Proc Natl Acad Sci U S A. 2009;106(48):20180-5.

21. Zhang X, Jantama K, Moore JC, Shanmugam KT, Ingram LO. Production of $\mathrm{L}$-alanine by metabolically engineered Escherichia coli. Appl Microbiol Biotechnol. 2007;77(2):355-66.

22. Patel RK, Jain M. NGS QC Toolkit. a toolkit for quality control of next generation sequencing data. PLoS One. 2012;7(2):e30619.

23. Langmead B, Salzberg SL. Fast gapped-read alignment with Bowtie 2. Nat Methods. 2012;9(4):357-9.

24. Soneson C, Delorenzi M. A comparison of methods for differential expression analysis of RNA-seq data. BMC Bioinformatics. 2013;14:91.

25. Mclntyre LM, Lopiano KK, Morse AM, Amin V, Oberg AL, Young LJ, Nuzhdin SV. RNA-seq: technical variability and sampling. BMC Genomics. 2011;12:293.

26. Anders S, Huber W. Differential expression analysis for sequence count data. Genome Biol. 2010;11(10):R106.

27. Ruepp A, Zollner A, Maier D, Albermann K, Hani J, Mokrejs M, Tetko I, Guldener U, Mannhaupt G, Munsterkotter M, et al. The FunCat, a functional annotation scheme for systematic classification of proteins from whole genomes. Nucleic Acids Res. 2004;32(18):5539-45 (http://mips.helmholtzmuenchen.de/funcatDB/).

28. Stoebel DM, Hokamp K, Last MS, Dorman CJ. Compensatory evolution of gene regulation in response to stress by Escherichia coli lacking RpoS. PLoS Genet. 2009;5(10):e1000671.

29. Becker LA, Cetin MS, Hutkins RW, Benson AK. Identification of the gene encoding the alternative sigma factor sigmaB from Listeria monocytogenes and its role in osmotolerance. J Bacteriol. 1998;180(17):4547-54.

30. Hwang S, Jeon B, Yun J, Ryu S. Roles of RpoN in the resistance of Campylobacter jejuni under various stress conditions. BMC Microbiol. 2011:11(1):1-8.

31. Schultz J, Milpetz F, Bork P, Ponting CP. SMART, a simple modular architecture research tool: identification of signaling domains. Proc Nat Acad Sci U S A. 1998;95:5857-64 (http://smart.embl.de/).

32. Darst SA, Polyakov A, Richter C, Zhang G. Structural studies of Escherichia coli RNA polymerase. Cold Spring Harb Symp Quant Biol. 1998;63(63):269-76.

33. Kristich CJ, Little JL. Mutations in the beta subunit of RNA polymerase alter intrinsic cephalosporin resistance in Enterococci. Antimicrob Agents Chemother. 2012;56(4):2022-27.

34. Helling RB. Why does Escherichia coli have two primary pathways for synthesis of glutamate? J Bacteriol. 1994;176(15):4664-8.

35. Hernandez PE, Ordonez JA, Perez BS. Use of the Mud(Ap, Lac) Bacteriophage to Study the Regulation of L-Proline Biosynthetic Genes in Escherichia coli K12. Curr Microbiol. 1983:9(1):31-5.

36. May G, Faatz E, Villarejo M, Bremer E. Binding protein dependent transport of glycine betaine and its osmotic regulation in Escherichia coli K12. Mol Gen Genet. 1986;205(2):225-33.

37. Bukau B, Ehrmann M, Boos W. Osmoregulation of the maltose regulon in Escherichia coli. J Bacteriol. 1986;166(3):884-91.

38. Gosset G. Improvement of Escherichia coli production strains by modification of the phosphoenolpyruvate:sugar phosphotransferase system. Microb Cell Fact. 2005;4(1):14.

39. Kaeriyama M, Machida K, Kitakaze A, Wang H, Lao Q, Fukamachi T, Saito H, Kobayashi H. OmpC and OmpF are required for growth under hyperosmotic stress above pH 8 in Escherichia coli. Lett Appl Microbiol. 2006:42(3):195-201.

40. Death A, Notley L, Ferenci T. Derepression of LamB protein facilitates outer membrane permeation of carbohydrates into Escherichia coli under conditions of nutrient stress. J Bacteriol. 1993;175(5):1475-83.

41. Tan Z, Zhu X, Chen J, Li Q, Zhang X. Activating phosphoenolpyruvate carboxylase and phosphoenolpyruvate carboxykinase in combination for improvement of succinate production. Appl Environ Microbiol. 2013; 79(16):4838-44. 
42. Kotrba P, Inui M, Yukawa H. Bacterial phosphotransferase system (PTS) in carbohydrate uptake and control of carbon metabolism. J Biosci Bioeng. 2001;92(6):502-17.

43. Postma PW, Lengeler JW, Jacobson GR. Phosphoenolpyruvate:carbohydrate phosphotransferase systems of bacteria. Microbiol Rev. 1993;57(3):543-94.

44. Wang Q, Wu C, Chen T, Chen X, Zhao X. Expression of galactose permease and pyruvate carboxylase in Escherichia coli ptsG mutant increases the growth rate and succinate yield under anaerobic conditions. Biotechnol Lett. 2006;28(2):89-93.

45. Todt JC, McGroarty EJ. Acid pH decreases OmpF and OmpC channel size in vivo. Biochem Biophys Res Commun. 1992;189(3):1498-502.

46. Sun L, Zeng X, Yan C, Sun X, Gong X, Rao Y, Yan N. Crystal structure of a bacterial homologue of glucose transporters GLUT1-4. Nature. 2012; 490(7420):361-6.

47. Schlegel A, Bohm A, Lee SJ, Peist R, Decker K, Boos W. Network regulation of the Escherichia coli maltose system. J Mol Microbiol Biotechnol. 2002:4(3):301-7.

48. Edgar R, Domrachev M, Lash AE. Gene Expression Omnibus: NCBI gene expression and hybridization array data repository. Nucleic Acids Res. 2002; 30:207-10. https://www.ncbi.nlm.nih.gov/geo/.

Submit your next manuscript to BioMed Central and we will help you at every step:

- We accept pre-submission inquiries

- Our selector tool helps you to find the most relevant journal

- We provide round the clock customer support

- Convenient online submission

- Thorough peer review

- Inclusion in PubMed and all major indexing services

- Maximum visibility for your research

Submit your manuscript at www.biomedcentral.com/submit
Biomed Central 